\title{
Uso de psyllium para controle de constipação em cães
}

\author{
The use of psyllium to control constipation in dogs
}

\author{
Letícia Tortola ${ }^{\mathrm{I}}$ Márcio Antonio Brunetto ${ }^{\mathrm{I}}$ Leandro Zaine ${ }^{\mathrm{I}}$ Ricardo Souza Vasconcellos ${ }^{\mathrm{II}}$ \\ Michele Cristina de Camargo Oliveira ${ }^{\mathrm{I}}$ Sandra Prudente Nogueira ${ }^{\mathrm{I}}$ \\ Aulus Cavalieri Carciofi ${ }^{\text {II }}$
}

\begin{abstract}
- NOTA -
\section{RESUMO}

Psyllium (Plantago psyllium) é um vegetal comumente utilizado como adjuvante no tratamento de afecções que resultam em constipação. Com o objetivo de melhor compreender os efeitos dessa fibra em cães, foram realizados dois experimentos. O primeiro avaliou, em cães sadios, inclusões de $0 \%, 2 \%$ e $4 \%$ de semente integral moída de psyllium à dieta (com base na matéria natural). Para tanto, foram empregados oito cães da raça Beagles adultos, os quais receberam as dietas experimentais por oito dias. Verificou-se aumento linear da umidade das fezes com a adição de teores crescentes de psyllium $\left(R^{2}=0,54\right.$ e $\left.P=0,0012\right)$, sem alteração do escore fecal e número de defecações por dia. O segundo experimento incluiu avaliação da opinião de 24 proprietários cujos cães receberam suplementação de $2 \%$ de psyllium como parte do tratamento de afecções que causaram constipação. Dos proprietários incluídos, 19 (79,2\%) relataram melhora da defecação de seus cães durante a administração da fibra. Quinze (62,5\%) observaram que as fezes dos animais, antes ressecadas ou endurecidas, tornaram-se normais ou pastosas.

ABSTRACT

Psyllium (Plantago psyllium) is a fiber generally used as a supplement in the treatment of diseases that led to constipation. To understanding of the effects of this fiber in dogs, two experiments were conducted. The first evaluated in healthy dogs a diet supplementation with $0 \%, 2 \%$ and $4 \%$ of ground whole psyllium seeds (as-fed basis). Eight adult Beagle dogs were used. They received the experimental diets for eight days. A linear increase of faecal moisture was verified with the addition of psyllium $\left(R^{2}=0.54\right.$ and $\left.P=0.0012\right)$, with no alteration on faecal score or number of defecations per day. The second experiment included 24 dogs with constipation. The dogs received a diet supplemented with $2 \%$ of psyllium as part of the treatment, and the owners were invited to answer a questionnaire. Nineteen (79.2\%) owners reported that their dog had an easier defecation process during supplementation. Fifteen (62.5\%) reported that the animals' faeces, which were dry or hard at the start, became normal or pasty after psyllium consumption. The results confirmed a laxative effect of psyllium in dogs, which was already reported in human medicine, supporting the use of this fiber in the clinical management of diseases that cause constipation in dog.
\end{abstract} Os resultados comprovaram para cães os efeitos laxativos do psyllium já descritos na medicina humana, indicando seu uso no tratamento de suporte de enfermidades que levam à constipação.

Palavras-chave: Plantago psyllium, cão, defecação, fibra solúvel, fezes.
Key words: Plantago psyllium, dog, defecation, soluble fiber, faeces.

O psyllium (Plantago psyllium) é um vegetal comumente utilizado como adjuvante no

IPrograma de Pós-graduação em Medicina Veterinária (PPGMV), área de concentração Clínica Médica, Faculdade de Ciências Agrárias e Veterinárias (FCAV), Universidade Estadual Paulista (Unesp), Jaboticabal, SP, Brasil.

"Pós-doutoramento em Medicina Veterinária, FCAV/Unesp, Jaboticabal, SP, Brasil.

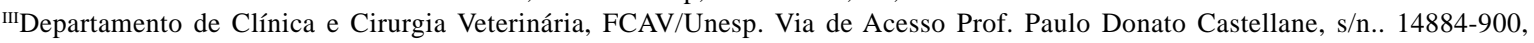
Jaboticabal, SP, Brasil. E-mail: aulus.carciofi@gmail.com. Autor para correspondência. 
tratamento de diversas enfermidades que resultam em alteração na eliminação de fezes, tanto em animais, como no homem. Dentre as indicações de seu uso, inclui-se constipação, diarreia, síndrome do intestino irritável, doença inflamatória intestinal, colite ulcerativa, neoplasias em cólon, diabetes mellitus e hipercolesterolemia (SINGH, 2007). Seu emprego em pacientes com constipação é bem difundido na medicina humana (BRANDT et al., 2005, RAMKUMAR \& RAO, 2005, JOHANSON, 2007). Porém, estudos sobre essa fibra são menos frequentes na medicina veterinária.

Trata-se, juntamente com a goma guar e a goma xantana, de fibra dietética altamente solúvel (DIKEMAN et al., 2006). Sua elevada solubilidade, aliada a sua baixa fermentabilidade no intestino grosso (MARLETT \& FISCHER, 2003), possibilita seu emprego em situações de alteração do trânsito gastrintestinal. Atribui-se ao psyllium efeito gelificante, retendo água no bolo fecal e favorecendo a normalização da taxa de passagem da digesta e a formação de fezes no cólon (SINGH, 2007).

O termo constipação é definido como defecação infrequente, com fezes excessivamente ressecadas ou endurecidas, comumente acompanhada por tenesmos. É um sinal clínico, não uma doença, e várias afecções podem causá-la (REMILLARD et al., 2000). Condições em cães que podem levar à constipação incluem: hérnias perineais, fraturas pélvicas ou em coluna lombo-sacra, aumento prostático e neoplasias retais (GERMAN \& ZENTEK, 2006). O tratamento médico convencional da constipação baseiase no uso de laxantes osmóticos (lactulose), surfactantes (citrato de sódio), lubrificantes (óleo mineral, parafina líquida), estimulantes (supositório de glicerina), drogas pró-cinéticas (cisaprida, ranitidina, tegaserod) e enema (água morna, glicerina, fosfato de sódio) (GERMAN \& ZENTEK, 2006). O uso de laxantes osmóticos, os medicamentos mais amplamente empregados, requer alguns dias até o efeito laxativo, podendo acarretar como efeitos adversos dor abdominal e flatulência (JOHANSON, 2007).

Dessa forma, a inclusão de fibra alimentar na dieta de cães apresenta-se como alternativa terapêutica interessante e natural, com possibilidade de emprego por longo prazo, com baixa perspectiva de ocorrência de efeitos colaterais. Assim sendo, com este estudo objetivou-se avaliar o emprego de semente integral moída de psyllium como alternativa terapêutica no manejo clínico de cães com constipação.

Experimento 1 - Esse ensaio teve por objetivo verificar o efeito da adição de semente integral moída de psyllium ${ }^{a}$ na formação das fezes de cães adultos sadios. Foi conduzido no Laboratório de Pesquisa em Nutrição e Doenças Nutricionais de Cães e Gatos "Prof. Dr. Flávio Prada” do Departamento de Clínica e Cirurgia Veterinária da Faculdade de Ciências Agrárias e Veterinárias da Unesp, campus de Jaboticabal. Foram empregados oito cães da raça Beagle adultos (quatro machos e quatro fêmeas, peso $9,7 \pm 1,2 \mathrm{~kg}$, idade $3,7 \pm 0,97$ anos), alojados em gaiolas metabólicas em inox $(90 \mathrm{~cm} x 90 \mathrm{~cm} x 90 \mathrm{~cm})$. Os cães foram alimentados com dieta comercial seca do tipo premium (Sabor e Vida Cães Adultos, Mogiana Alimentos S. A., Campinas, SP) em quantidade suficiente para atender sua necessidade de energia metabolizável (NRC, 2006). A quantidade total diária de alimento foi fornecida em duas refeições, e água ficou disponível durante todo o período experimental.

O ensaio durou oito dias. Nos dias um a quatro, os oito cães foram alimentados apenas com a dieta comercial, sem adição de psyllium, quando os resultados de produção de fezes representaram o grupo controle. Nos dias cinco a oito, os cães foram divididos em dois grupos de quatro animais cada um, quando um grupo de quatro cães passou a receber, em adição à dieta comercial, $2 \%$ de semente integral moída de psyllium e o outro grupo, em adição à dieta comercial, $4 \%$ de semente integral moída de psyllium. A suplementação era aplicada por sobre a refeição do animal, com a ração já em seu comedouro. A suplementação de psyllium foi calculada com base na quantidade de alimento fornecida ao animal, pela seguinte fórmula:

Suplementação de psyllum (g) = Alimento consumido (g) $x$ \% adição $^{1}$

100

1-porcentagem de adição de $2 \%$ ou $4 \%$, a depender do grupo experimental.

As fezes dos cães foram coletadas duas vezes ao dia. Estas tiveram seu escore determinado, sendo então pesadas e acondicionadas em sacos plásticos individuais, previamente identificados, fechados e armazenados em freezer $\left(-20^{\circ} \mathrm{C}\right)$. Ao final do período de coleta, as fezes foram descongeladas e homogeneizadas, compondo-se uma amostra por animal e período. Estas foram pré-secas em estufa de ventilação forçada (320-SE, FANEM, São Paulo - SP), a $55^{\circ} \mathrm{C}$, durante 72 horas. As fezes pré-secas foram moídas em moinho tipo faca(MOD 340, ART LAB, São Paulo - SP), com peneira de $1 \mathrm{~mm}$, para posterior determinação de seu teor de matéria seca a $105^{\circ} \mathrm{C}$ (AOAC, 1995). Foram quantificados, também, o número de defecações por animal por dia e a qualidade das fezes por meio do escore (CARCIOFI et al., 2008), atribuindo-se graduações de 0 a 5 , sendo: 0 para fezes 
líquidas; 1 fezes pastosas e sem forma; 2 para fezes macias, mal-formadas e que assumem o formato do recipiente de colheita; 3 para fezes macias, formadas, úmidas e que marcam o piso; 4 para fezes bem formadas, consistentes e que não marcam o piso; 5 para aquelas também bem formadas, mas duras e secas. Consideramse normais os valores entre 3 e 4 .

O estudo seguiu delineamento inteiramente casualizado. Os dados paramétricos (umidade fecal e número de defecações) foram avaliados pelo Teste $\mathrm{F}$ $(\mathrm{P}<0,05)$. Análise de regressão linear foi empregada para avaliar os níveis de inclusão de psyllium $(0 \%, 2 \%$ ou $4 \%)$ e a umidade das fezes dos cães $(\mathrm{P}<0,05)$. O escore fecal foi comparado pelo teste de Kruskal-Wallis para dados nãoparamétricos $(\mathrm{P}<0,05)$. Todas as análises foram realizadas no software estatístico SAS ${ }^{\mathrm{b}}$ (1990).

Todos os cães consumiram adequadamente a dieta e o suplemento, sem episódios de recusa alimentar, vômito ou diarreia. Verificou-se aumento linear $\left(r^{2}=0,54 ; \mathrm{P}=0,005\right)$ no teor de umidade das fezes (média \pm erro padrão) ao se comparar o grupo controle $(60,63 \pm 1,52)$ com o grupo suplementado com psyllium a $2 \%(62,81 \pm 1,91)$ e $4 \%(68,40 \pm 0,78)$. Não houve diferença no escore fecal $(3,95 \pm 0,06 ; \mathrm{P}>0,05)$, nem no número de defecações por cão por dia $(1,29 \pm 0,13$; $\mathrm{P}>0,05)$.

Experimento 2 - Esse ensaio teve por objetivo verificar o efeito da suplementação de psyllium em cães com constipação. Foram selecionados 24 cães atendidos entre os meses de abril e dezembro de 2008 no Hospital Veterinário da Faculdade de Ciências Agrárias e Veterinárias da Unesp, campus de Jaboticabal. Em todos os pacientes, empregou-se suplementação de $2 \%$ de psyllium à dieta como parte do tratamento de afecções que causaram ou predispunham à constipação. Os proprietários desses animais foram interrogados, preenchendo-se um questionário estruturado sobre a resposta dos animais ao tratamento com a fibra. Foram excluídos do estudo todos os cães que, além de psyllium, necessitaram receber outros fármacos ou produtos que pudessem interferir nos resultados obtidos.

Dos cães incluídos no estudo, 11 (45,8\%) apresentavam hérnia perineal como afecção primária levando à disquesia, cinco (20,8\%) apresentaram fratura de pelve, dois $(8,3 \%)$ apresentaram afecção em coluna vertebral, um $(4,2 \%)$ apresentou aumento prostático, um $(4,2 \%)$ apresentou hérnia inguinal, um (4,2\%) apresentou pólipos inflamatórios em reto, um $(4,2 \%)$ apresentou inflamação da glândula adanal, um $(4,2 \%)$ apresentou nódulo perianal e um (4,2\%) apresentou miíase em região anal. Em resposta ao questionário, a frequência de administração do psyllium foi de uma vez ao dia em dois (8,3\%) cães, duas vezes em 20 $(83,3 \%)$, três vezes em um $(4,2 \%)$ e quatro vezes ao dia em um (4,2\%) animal. A consistência das fezes antes da suplementação era pastosa em dois (8,3\%) cães, normal em cinco (20,8\%), ressecada em seis (25\%) e endurecida em 11 (45,8\%) animais. Durante o uso de psyllium, as fezes foram consideradas pastosas por parte de quatro (16,7\%) proprietários, normais por 18 (75\%) e ressecadas por dois $(8,3 \%)$ dos proprietários interrogados, de forma que 15 (62,5\%) proprietários observaram que as fezes dos animais, antes ressecadas ou endurecidas, tornaram-se normais ou pastosas. Por fim, em relação ao ato de defecação, cinco (20,8\%) proprietários não notaram nenhuma melhora, três $(12,5 \%)$ relataram pouca melhora, três (12,5\%) relataram melhora razoável e 13 (54,2\%) relataram importante melhora, com maior facilidade de defecar por parte de seus cães. Nenhum proprietário relatou recusa do animal ingerir o alimento ou quaisquer distúrbios gastrintestinais durante sua ingestão.

Em estudo recente, CALABRÒ et al. (2008) avaliaram a fermentação in vitro de 11 substratos incubados em inóculo fecal de cão, incluindo a amostra de semente moída de psyllium empregada no presente estudo. Observaram que o psyllium demonstrou, após 48h de incubação, 28,8\% de desaparecimento da matéria orgânica, valor inferior ao de fibras altamente fermentáveis, como inulina, frutoligossacárides e farelo de trigo. Os autores verificaram, ainda, mais lenta produção de ácidos graxos de cadeia curta $\left(\mathrm{mL} \mathrm{h}^{-1}\right)$, mas com intermediária produção total $\left(\mathrm{mmol} \mathrm{g}^{-1}\right)$. O psyllium, quando dissolvido em água, aumenta de oito a 14 vezes seu tamanho original, conferindo viscosidade à solução elevada (DIKEMAN, et al., 2006). Acredita-se que essa gelificação do bolo alimentar promova retenção de água na luz intestinal e aumento do peristaltismo, explicando a hidratação das fezes verificada com seu consumo no experimento 1 .

Como resultado da retenção de água e produção de ácidos graxos de cadeia curta, essa fibra apresenta efeito laxativo para o homem, com amolecimento das fezes, colaborando no controle da constipação crônica (SINGH, 2007), fato também confirmado para cães no experimento 2 . A verificação de um efeito dose-resposta no teor de umidade das fezes sugere, ainda, que outros teores de inclusão possam resultar em maiores retenções de água no bolo fecal, com possível maior amolecimento das fezes. Apesar de essa hipótese não ter sido avaliada no presente estudo, sugere ser possível ao clínico veterinário variar a dose de psyllium administrada ao paciente, de acordo com as condições clínicas e respostas individuais de cada animal. 
Os resultados aqui encontrados confirmam para cães o efeito laxativo do psyllium já descrito em medicina humana, indicando o uso dessa fibra no tratamento suporte das enfermidades que levam à constipação. A forma de cálculo proposta no presente trabalho para a prescrição do psyllium, estimada juntamente com a alimentação dos cães ao invés de ser estabelecida em função do peso corporal dos animais, demonstrou ser segura e confiável.

\section{AGRADECIMENTOS}

À Mogiana Alimentos S.A. (Guabi), pelo fornecimento da ração durante o experimento e pelo apoio financeiro na forma de concessão de Bolsa de Aprimoramento para a primeira autora; à Dilumix Industrial Ltda., pela doação do psyllium utilizado no estudo.

\section{FONTES DE AQUISIÇÃO}

a-Semente integral moída de psyllium. Composição: fibra dietética total (FDT) 51\%, fibra dietética insolúvel (FDI) 40,1\% e fibra dietética solúvel (FDS) 10,9\%.

b-Statistical Analysis Systems Institute Inc., 1997. SAS system for elementary statistical analysis. 2nd ed. Cary, NC, USA.

\section{REFERÊNCIAS}

AOAC. Association of official analytical chemists. Official methods of analysis. 16.ed. Washington, DC, 1995. Chap.4, p.1-45.

BRANDT, L.J. et al. Systematic review on the management of chronic constipation in North America American. Journal of Gastroenterology, v.100, supl.1, p.05-21, 2005. Disponível em: $<$ http://www.nature.com/ajg/journal/v100/n1s/full/ ajg2005520a.html $>$. Acesso em: 02 set. 2009. doi: 10.1111/j.15720241.2005.50613.x.

CALABRÒ S. et al. In vitro evaluation of different fiber sources and potential set prebiotics for dogs. In: CONGRESS OF THE EUROPEAN SOCIETY OF VETERINAY AND COMPARARATIVE NUTRITION, 12., 2008, Vienna, Austria. Anais... Vienna: University of Veterinary Medicine Vienna, 2008. V.1, 154p, p.63.

CARCIOFI, A.C. et al. Effects of six carbohydrate sources on dog diet digestibility and post-prandial glucose and insulin response. Journal of Animal Physiology and Animal Nutrition, v.92, p.326-336, 2008. Disponível em: <http:// www3.interscience.wiley.com/journal/119408472/ abstract?CRETRY=1\&SRETRY=0>. Acesso em: 02 set. 2009. doi: 10.1111/j.1439-0396.2007.00794.x.
DIKEMAN, C.L. et al. Dietary fibers affect viscosity of solutions and simulated human gastric and small intestinal digesta. Journal of Nutrition, v.136, p.913-919, 2006. Disponível em: <http://jn.nutrition.org/cgi/content/full/136/4/ 91>3. Acesso em: 02 set. 2009.

GERMAN, A.; ZENTEK, J. The most common digestive diseases: the role of nutrition. In: PIBOT, P. et al. Encyclopedia of canine clinical nutrition. Airmargues: Aniwa SAS, 2006. p.92-133. Disponível em: <http http:// www.ivis.org/advances/rc/chap03part02/chapter.asp?LA=1> . Acesso em: 02 set. 2009.

JOHANSON, J.F. Review of the treatment options for chronic constipation. Medscape General Medicine, v.9, n.2, p.25, 2007. Disponível em: <http://www.pubmedcentral.nih.gov/ articlerender.fcgi?artid=1994829>. Acesso em: 02 set. 2009.

MARLETT, J.A.; FISCHER, M.H. The active fraction of psyllium seed husk. Proccedings of the Nutrition Society, v.62, n.1, p.207209, 2003 . Disponível em: <http:// j o u r n a l s. c a m b r id g e. or g/a c t i o n / displayFulltext type $=6 \& \mathrm{fid}=807288 \& \mathrm{jid}=\&$ volumeId $=\&$ issue Id $=01 \&$ a id $=807284 \&$ b ody Id $=\&$ me mbersh ip Nu m ber=\&societyETOCSession=\&fulltextType=MR\&fileId=S0029665103000326 $>$. Acesso em: 02 set. 2009. doi: 10.1079/PNS2002201.

NRC. National Research Council. Nutrient requirements of dogs and cats. Whashington, DC: The National Academy, 2006. 398p.

SINGH, B. Psyllium as therapeutic and drug delivery agent. International Journal of Pharmaceutics, v.334, n.1-2, p.114, 2007. Disponível em: <http://www.sciencedirect.com/ science? ob=ArticleURL \& udi=B6T7W-4MW3215$2 \& \_$user $=972052 \& \_$rdoc $=1 \& \_\mathrm{fmt}=\& \_$orig $=$search $\& \_s o r t=\mathrm{d} \& \_d$ ocanchor $=\& v i e w=c \& \_s e a r c h S t r I d=998458432 \& \_$rerunOrigin $=$go ogle\&_acct $=\mathrm{C} 000049647 \&$ \& version $=1 \&$ \&urlVersion $=0$ \&_userid=972052\&md5=ca46d8f39e3b77f9baba99d9470e2a77 > . Acesso em: 02 set. 2009. doi: 10.1016/j.ijpharm.2007.01.028.

RAMKUMAR, D.; RAO, S.S.C. Efficacy and safety of traditional medical therapies for chronic constipation: systematic review. American Journal of Gastroenterology, v.100, n.4, p.936-971, 2005. Disponível em: <http:// www.nature.com/ajg/journal/v100/n4/abs/ajg2005161a.html> . Acesso em: 02 set. 2009. doi: 10.1111/j.15720241.2005.40925.x.

REMILLARD, R.L. et al. Gastrointestinal and exocrine pancreatic disease. In: HAND, M.S. et al. Small animal clinical nutrition. 4.ed. Topeka: Mark Morris Institute, 2000. p.725-810. 